\title{
Working and Limitations of Cable Stiffening in Flexible Link Manipulators
}

\author{
Rahul Dixit ${ }^{1}$ and R. Prasanth Kumar ${ }^{2}$ \\ ${ }^{1}$ Control Systems Laboratory, Research Center Imarat, Vigyanakancha, Hyderabad 500069, India \\ ${ }^{2}$ Department of Mechanical \& Aerospace Engineering, Indian Institute of Technology Hyderabad, Kandi, Telangana 502285, India
}

Correspondence should be addressed to R. Prasanth Kumar; rpkumar@iith.ac.in

Received 30 April 2016; Revised 30 June 2016; Accepted 14 July 2016

Academic Editor: Emil Manoach

Copyright ( 2016 R. Dixit and R. P. Kumar. This is an open access article distributed under the Creative Commons Attribution License, which permits unrestricted use, distribution, and reproduction in any medium, provided the original work is properly cited.

Rigid link manipulators (RLMs) are used in industry to move and manipulate objects in their workspaces. Flexible link manipulators (FLMs), which are much lighter and hence highly flexible compared to RLMs, have been proposed in the past as means to reduce energy consumption and increase the speed of operation. Unlike RLM, an FLM has infinite degrees of freedom actuated by finite number of actuators. Due to high flexibility affecting the precision of operation, special control algorithms are required to make them usable. Recently, a method to stiffen FLMs using cables, without adding significant inertia or adversely affecting the advantages of FLMs, has been proposed as a possible solution in a preliminary work by the authors. An FLM stiffened using cables can use existing control algorithms designed for RLMs. In this paper we discuss in detail the working principle and limitations of cable stiffening for flexible link manipulators through simulations and experiments. A systematic way of deciding the location of cable attachments to the FLM is also presented. The main result of this paper is to show the advantage of adding a second pair of cables in reducing overall link deflections.

\section{Introduction}

Robot manipulators used in industry spend much of their energies in moving their end effectors from one point to another. Their links are designed to have very low deflection which makes them bulky. Hence they are called rigid link manipulators (RLMs). In order to reduce wastage of energy in moving the manipulator link mass, researchers proposed flexible link manipulators (FLMs) (Figure 1) which are not only much lighter but also highly flexible because of the reduced inertia. Moving a payload at the end effector of FLM to desired location at the specified time requires special control algorithms designed taking into account the underactuated nature of FLMs.

In order to minimize the tip vibration of FLMs, several researchers have analyzed the problem in different ways. Having a more accurate model helps in better control of FLM. Some researchers have focused on modeling of the flexible arm [1-8] so as to control it effectively. Modeling of the flexible arm has been studied for single $[2,6,7]$ as well as multiple flexible link systems [4]. Piedboeuf [9] presented six methods to model a rotating flexible beam considering foreshortening effect. Sugiyama et al. [10] presented finite segment modeling of flexible link considering discrete segments. The method explains modeling of finite segments using torsional springs between adjacent segments. Kiran et al. [11] presented bond graph technique for modeling a single link flexible space manipulator. Kinematic analysis of the FLM has been studied and suitability of coordinate frame was discussed in [8].

Different methods of control to reduce or eliminate tip oscillations of FLM have been studied which includes resonant control [12], sliding mode control [13-16], optimal control strategies [2], and other nonlinear control methods [17]. Morales et al. [18] studied behaviours of light weight single link FLM with payload variations by using disturbance observer. Baroudi et al. [19] presented their study on control of flexible manipulator using LQR technique. Ahmad and Mohamed [20] compared the control performance of LQR and PID to suppress the tip vibration. Several authors have used closed loop control methodologies by modeling flexible 


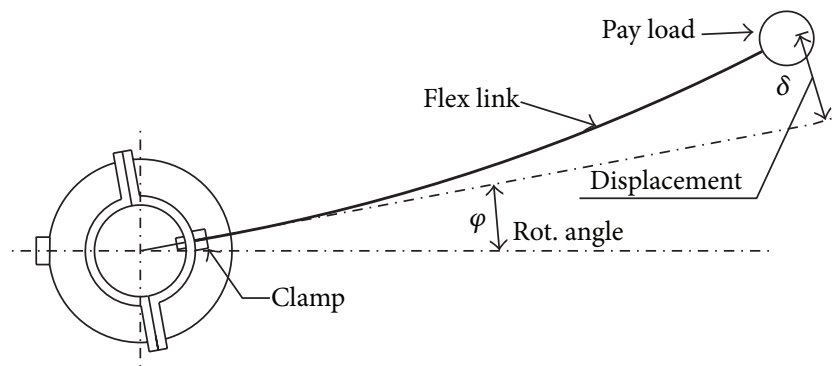

FIGURE 1: Schematic of flexible link manipulator (FLM).

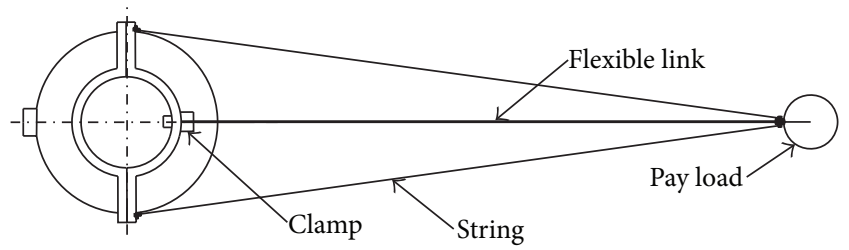

Figure 2: Schematic of cable stiffened FLM.

link and using sensors along the flexible link for closing the control loop. The closed loop control requires several feedback sensors and complex control algorithms. Usage of these sensors and complex control algorithms further increase the cost of system. In addition to that the accuracy of control becomes dependent on the sensor accuracy as well as the variation in control parameters. Researchers have also studied command shaping to reduce tip oscillations [21-26]. Further, force interaction with environment using FLMs remains an open problem. It has been observed that the control philosophy used for rigid link manipulators cannot be applied to FLMs directly. Despite research into special control algorithms for FLMs for more than two decades, they have not yet been adopted by industry.

Stiffening an FLM using a pair of cables attached between the flexible link and its rigid hub as shown in Figure 2 has recently been proposed by Dixit and Prasanth Kumar [27] as possible means of overcoming the disadvantages of FLMs. Mass of cables for stiffening are very small compared to the mass of FLM itself. Hence they do not add significantly to the inertia of the manipulator. An FLM so stiffened can use the same control algorithms used for RLMs. Although the tip oscillations of cable stiffened FLM are minimized, radial component of force that occurs during acceleration and deceleration is found to cause buckling-like deflection in the middle of the FLM.

In this paper we focus on quantitatively showing how a pair of cables attached to the tip of an FLM increases the fundamental natural frequency and thus stiffens the FLM. This is done through numerical simulations in ANSYS software as well as experimental data. Further, the cause of buckling-like mid-link deflection when one pair of cables are used for stiffening is investigated analytically. It will be shown that adding a second pair of cables to the same rigid hub will further increase the fundamental frequency making the FLM stiffer. A systematic way of deciding the location of cable attachment on the FLM is also discussed.

\section{Mathematical Model and Simulation}

The flexible link manipulator studied in the paper is modeled using finite segment method (also called rigid finite element method). This method is known to give good predictions even in the presence of contact forces, large translations, and rotations. The mathematical model of the FLM is similar to the one presented in Dixit and Prasanth Kumar [27]. However, since the current paper also deals with two pairs of cables, the following description is briefly repeated here for reference. The flexible link is discretized into a finite number of rigid links interconnected by revolute joints with torsional springs and dampers. The damping coefficients are usually obtained from experiments. Torsional spring constants are determined from flexural rigidity as follows:

$$
\begin{aligned}
K^{i(i-1)} & =\frac{E I}{L} \quad i=2, \ldots, N, \\
K^{1(0)} & =\frac{2 E I}{L}
\end{aligned}
$$

for the first torsional spring at the hub,

where $L$ is the length of each of $N$ segments.

Hub is considered as the body- 0 and payload as body$(N+1)$, with $N$ rigid segments connecting the hub and payload. Therefore, there are $N+2$ bodies in total for $N$ segment discretization of the flexible link. The outer end of $\mathrm{Nth}$ rigid segment is connected to the payload through a rigid joint. Since the cables from hub support are also connected at the same point, spring forces due to stiffness of the cables act at that point. Equations of motion of the planar multibody system can be written as follows:

$$
\mathbf{M} \ddot{\mathbf{q}}=\mathbf{h}+{ }^{(c)} \mathbf{h},
$$

where mass matrix $\mathbf{M}=\operatorname{diag}\left(\left[\begin{array}{lllll}\mathbf{M}_{0} & \mathbf{M}_{1} & \cdots & \mathbf{M}_{N} & \mathbf{M}_{N+1}\end{array}\right]\right)$, array of body coordinates $\mathbf{q}=\left[\begin{array}{lllll}\mathbf{q}_{0}^{T} & \mathbf{q}_{1}^{T} & \cdots & \mathbf{q}_{N}^{T} & \mathbf{q}_{N+1}^{T}\end{array}\right]^{T}$, array of applied forces $\mathbf{h}=\left[\begin{array}{lllll}\mathbf{h}_{0}^{T} & \mathbf{h}_{1}^{T} & \cdots & \mathbf{h}_{N}^{T} & \mathbf{h}_{N+1}^{T}\end{array}\right]^{T}$, and array of constraint forces ${ }^{(c)} \mathbf{h}=$ $\left[\begin{array}{lllll}{ }^{(c)} \mathbf{h}_{0}^{T} & { }^{(c)} \mathbf{h}_{1}^{T} & \ldots & { }^{(c)} \mathbf{h}_{N}^{T} & { }^{(c)} \mathbf{h}_{N+1}^{T}\end{array}\right]^{T}$.

The applied force vector $\mathbf{h}$ consists of all zeros, except for $\mathbf{h}_{N}$ and $\mathbf{h}_{s}$ which depend on cable stiffness and end deflection. While $\mathbf{h}_{N}$ is the applied force on the tip of the link due to first pair of cables, $\mathbf{h}_{s}$ is the force due to second pair of cables on body-s. At any point of time, either one of the cables is in tension applying transverse and longitudinal forces on the tip. Constraint forces are determined from Jacobian matrix and Lagrange multipliers. Apart from rigid joint constraints and revolute joint constraints on the bodies, an additional driver constraint on the hub specifies desired angular displacement trajectory as a 3-4-5 polynomial as follows:

$$
\phi_{0}(t)=a_{0}+a_{1} t+a_{2} t^{2}+a_{3} t^{3}+a_{4} t^{4}+a_{5} t^{5} .
$$


TABLE 1: Simulation parameters of FLM.

\begin{tabular}{lc}
\hline Parameter & Value \\
\hline Flexible link size & $500 \times 30 \times 1.5$ \\
Mass of link & $0.175 \mathrm{~kg}$ \\
Mass of clamp & $0.150 \mathrm{~kg}$ \\
Distance of cable attachment & $70 \mathrm{~mm}$ \\
from shaft axis & $0.18 \mathrm{~kg}, 0.12 \mathrm{~kg}, 0.06 \mathrm{~kg}$ \\
Mass of payload & $7800 \mathrm{~kg} / \mathrm{m}^{3}$ \\
Density of link & $0.8 \mathrm{~mm}$ \\
Diameter of cable & $1 \mathrm{pair}$ and $2 \mathrm{pairs}$ \\
Number of cables & $7800 \mathrm{~kg} / \mathrm{m}^{3}$ \\
Cable material density & $45 \mathrm{deg}$. \\
Target rotation & $1 \mathrm{sec}$ \\
Time duration & \\
\hline
\end{tabular}

Coefficients of this polynomial are determined from the following conditions:

$$
\begin{aligned}
& \phi_{0}(0)=0, \\
& \dot{\phi}_{0}(0)=0, \\
& \ddot{\phi}_{0}(0)=0, \\
& \phi_{0}(1)=\frac{\pi}{4}, \\
& \dot{\phi}_{0}(1)=0, \\
& \ddot{\phi}_{0}(1)=0 .
\end{aligned}
$$

Table 1 lists the parameter and values used for simulation. A damping coefficient of 0.01 is found to closely match the vibration decay observed in experiments on flexible link. The results of simulations are shown in Figures 3 and 4. Without cables, the tip deflection is quite high. Using one pair of cables attached between tip and hub, deflections are brought down to less than a millimeter. Although this may seem reasonably small deflection, it will be shown in the next section that this could lead to high mid-link deflection resulting in foreshortening.

\section{Deflection Analysis and Cable Attachment Location}

Analysis of mode shapes of a flexible link gives valuable information on the location of attachment of cables on the FLM to minimize tip vibration. FLMs in clamped-free condition will have maximum deflection at the free tip for its first normal mode. This is similar to a cantilever beam which has maximum deflection at the free end. Figure 5(a) shows the first and second normal modes of a cantilever beam. For the given smooth trajectory input, first normal mode is more prominent than the second normal mode. Therefore, the location of attachment of the first pair of cables will be at the tip. With this attachment at the tip resisting the tip

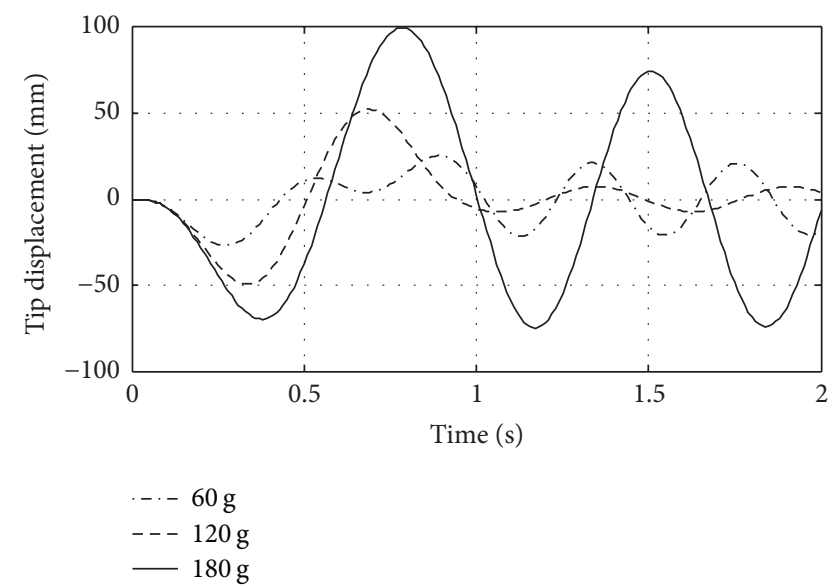

FIGURE 3: FLM tip deflection without cables for various payloads.

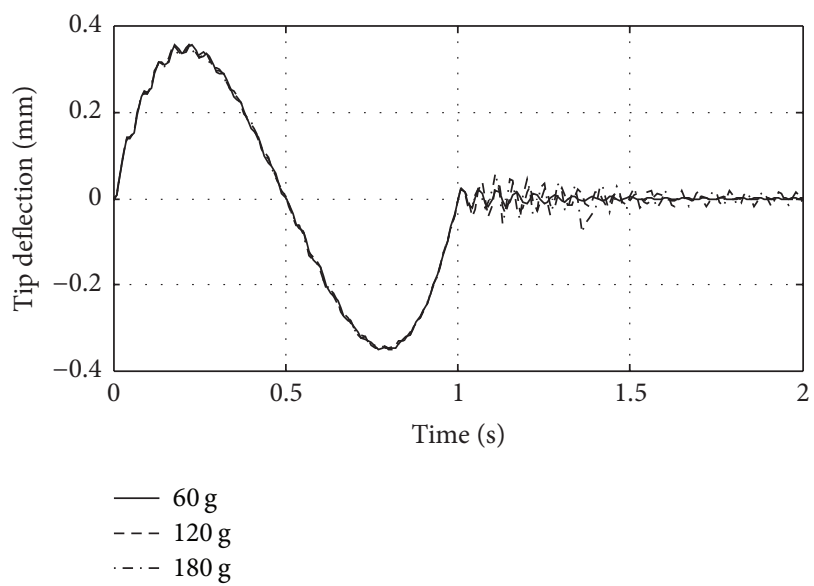

FIGURE 4: FLM tip deflection with one pair of cables for various payloads.

deflection, the flexible link will behave similar to a clampedpinned beam rather than a clamped-free beam. Figure 5(b) shows the normal mode shapes of a cantilever beam with the new clamped-pinned boundary condition.

It should be noted that the behavior of FLM with the first pair of cables will be similar to that of a cantilever beam with the end pinned but will not be identical. It is because a pinned joint does not allow translation in any direction whereas cable attachment does allow translation consistent with the constraints imposed by both cables. For this reason, we also try to validate the results of maximum deflection with multibody dynamics simulation and mode analysis through ANSYS simulation which are closer to the actual physical model.

After attachment of the first pair of cables, multibody dynamics analysis as described in previous section for an FLM with one pair of cables at the tip has been carried out for the one second trajectory in (3). For each time step, deflection of the FLM in local frame (attached at the hub of the FLM) with respect to the coordinate along its length has been obtained from simulation. At each time step there will be a deflection curve with maximum deflection at some point 


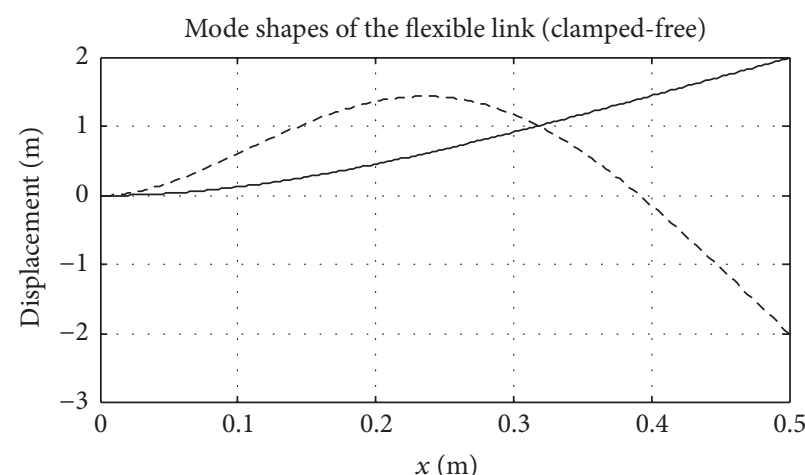

- First mode

--- Second mode

(a) Cantilever mode shapes for clamped-free boundary condition

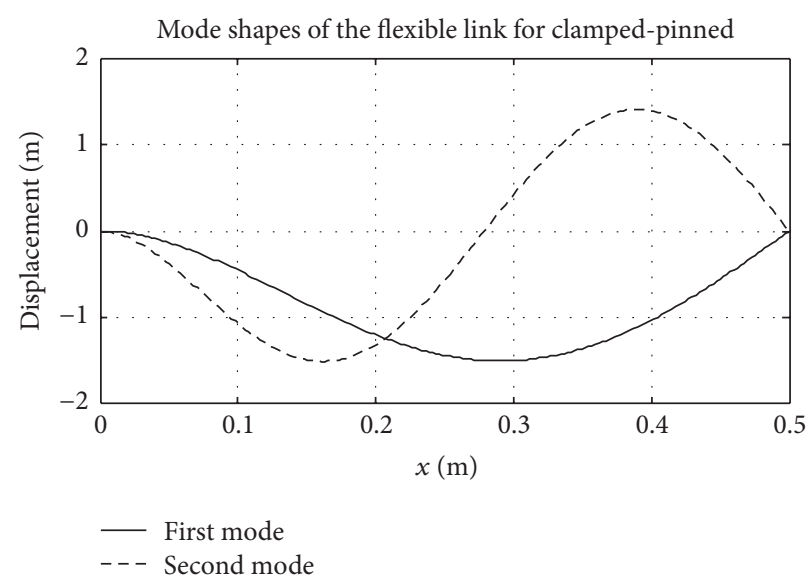

(b) Cantilever mode shapes for clamped-pinned boundary condition

FIgURE 5: Mode shapes of a cantilever beam with clamped-free and clamped-pinned boundary conditions.

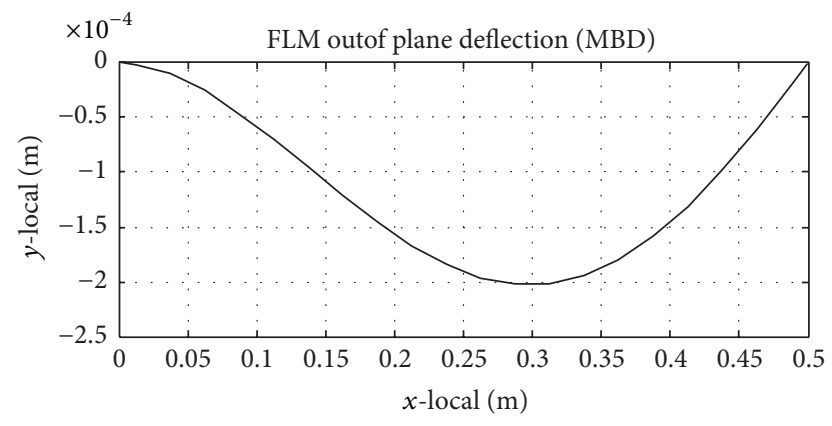

FIGURE 6: FLM maximum deflection curve with one pair of cables at the tip with 180 gram payload.

along the $x$-axis of the local frame. A deflection curve whose maximum deflection is larger than the maximum deflection at all other time steps is chosen. The location of maximum deflection would be the ideal location for the attachment of second pair of cables to minimize the maximum deflection. It can be seen from Figure 6 that the optimal location for second pair attachment can be at $0.3 \mathrm{~m}$ for an FLM of $0.5 \mathrm{~m}$ length. It is interesting to note that the FLM maximum deflection pattern resembles the first normal mode shape of a cantilever beam with one end fixed and the other end pinned plotted in Figure 5(b). It can be seen that the location of maximum deflection also matches very closely the multibody dynamics analysis. Therefore, it can be concluded that the optimal location for the attachment of the second pair of stiffening cables will be at $x=0.3 \mathrm{~m}$.

3.1. Buckling-Like Mid-Link Deflection. One of the important advantages of stiffened FLM is its fast speed of operation. Upper limit of acceleration of the FLM is limited by maximum bending moment on the link. The higher the acceleration or deceleration is, the more the FLM is prone to mid-link deflection (deflection between hub and tip). Figure 6 shows that the mid-link deflection is as high as $2 \mathrm{~mm}$. In the following analysis, we assume the link is in dynamic

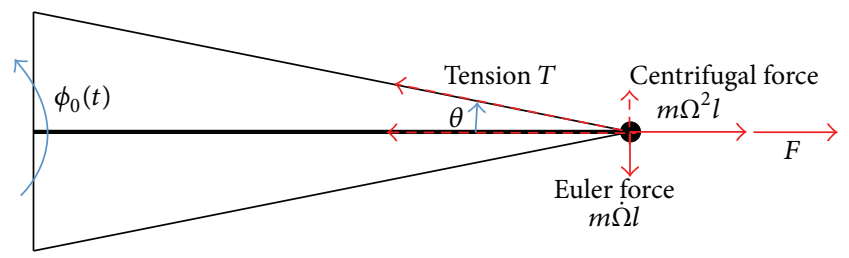

FIGURE 7: Forces acting on the payload at the tip of FLM.

equilibrium under constant angular acceleration, and the FLM mass is negligible compared to payload mass. Further, the radial buckling load can be assumed to be negligible since the FLM is thin and long. Force balance as shown in Figure 7 provides us the following set of equations:

$$
\begin{aligned}
m \Omega^{2} l-T \cos \theta & =F, \\
m \dot{\Omega} l & =T \sin \theta,
\end{aligned}
$$

where $T$ is tension in the cable, $\theta$ is the angle made by the cable with the FLM, $F$ is the net horizontal force on the payload in the local frame, and $\Omega=\dot{\phi}_{0}(t)$ is the angular velocity of the hub. The payload is subjected to centrifugal force along the radial direction away from the center and Euler force perpendicular to the radial direction and opposite to the direction of acceleration. Since the payload is assumed to be stationary in the local frame, there is no Coriolis force.

If $F$ is negative, the radial load on the link is compressive which is to be avoided. For no compressive load ( $F \geq 0$ or $m \Omega^{2} l \geq T \cos \theta$,

$$
\dot{\Omega} \leq \Omega^{2} \tan \theta .
$$

Equation (6) provides the condition for compressive tip load on the manipulator link. It can be observed that the abovementioned condition cannot be satisfied for any trajectory starting from rest and hence there will always be a compressive load on the link that causes mid-link deflection. If accelerations are allowed for short duration at the beginning 
and end of trajectory such that (6) is satisfied for most of the time of trajectory's duration, then these accelerations have to be higher than normal. In the extreme case, these sudden accelerations act as impact on the cables causing permanent deformation or even complete failure due to snapping. Permanent deformation increases the cable length, introduces slackness in the cable, and thus increases the tip deflection.

$\Omega^{2}$ term on the right-hand side of (6) is scaled by $\tan \theta$. The angle $\theta$ depends on the distance from motor axis to the point where cable is attached to the hub. For the same trajectory, the larger the value of $\theta$ is, the sooner the condition is satisfied. However, due to practical limitations, $\theta$ cannot be increased beyond a certain limit. For example, $\theta=45^{\circ}$ requires a hub as big as the length of the link itself on either side of the motor axis.

Hence, the simplest way to resist mid-link deflection due to acceleration and deceleration is to use a second pair of cables attached between the rigid hub and the ideal location discussed earlier. This solves the problem with only a negligible increase in the rotating inertia.

3.2. Buckling of FLM. It has been shown in Section 3.1 that after attachment of single pair of strings at the tip of FLM, the tip boundary condition can be taken as pinned; that is, displacement is very close to zero and the slope needs not be zero at the tip. Therefore, considering fixed-pinned boundary condition for the hub and tip, respectively, critical buckling load can be given by

$$
P_{\mathrm{cr}}=\frac{2 \pi^{2} E I}{l^{2}} .
$$

When a second pair of strings is attached at $0.6 l$ as discussed in Section 3, the attachment point can be assumed to act like a node. Figure 8 shows the configuration of FLM with two pairs of strings.

With reference to Figure 8, after attachment of second pair of string at $0.6 l$, the FLM is divided into two segments. Boundary conditions for segment- 1 of length $l_{1}$ will be fixedpinned and for segment- 2 of length $l_{2}$, it will be pinnedpinned. We write the equation for critical buckling load at the tip for full FLM and at the mid-link for segment-1 as follows:

$$
\begin{aligned}
& P_{\mathrm{cr} 1}=\frac{2 \pi^{2} E I}{l_{1}^{2}}, \\
& P_{\mathrm{cr} 2}=\frac{\pi^{2} E I}{l_{2}^{2}},
\end{aligned}
$$

where $l_{1}$ and $l_{2}$ are the lengths of segment- 1 and segment-2, respectively.

In order to have higher critical buckling load that allows higher accelerations to be used after the attachment of second pair of strings, the following conditions have to be satisfied:

$$
\begin{aligned}
& P_{\text {cr } 1}>P_{\text {cr }}, \\
& P_{\text {cr } 2}>P_{\text {cr }} .
\end{aligned}
$$

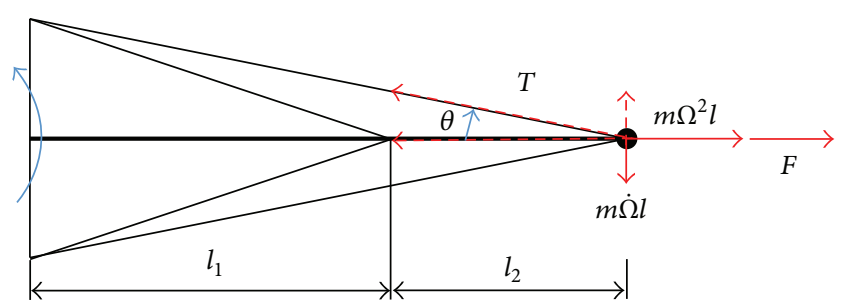

FIGURE 8: FLM with two pairs of strings.

For segment-1,

$$
\frac{2 \pi^{2} E I}{l_{1}^{2}}>\frac{2 \pi^{2} E I}{l^{2}} .
$$

Therefore, $l_{1}<l$, which is true as the second pair attachment location lies between hub and the tip.

Similarly, for the second segment,

$$
\frac{\pi^{2} E I}{l_{2}^{2}}>\frac{2 \pi^{2} E I}{l^{2}}
$$

Therefore, $l_{2}<0.707 l$, which is also true as $l_{2}=0.4 l$ as presented in Section 3.1.

Therefore, it can be concluded that the attachment of second pair of string enhances the buckling load thereby helping to achieve higher accelerations than achievable with a single pair of strings.

Another important conclusion derived from this analysis is that if the location of second pair attachment is at $0.293 \mathrm{l}$ from the hub, the critical buckling load due to attachment of second pair will not be higher than that of the critical buckling with only one pair of strings. Therefore, in order to have higher critical buckling load, second pair of strings should be attached beyond $0.293 \mathrm{l}$ from the hub. The location of attachment of second pair of strings proposed in this paper is at $0.6 l$.

3.3. Effect of Stiffening. In order to know the effect of cable attachment quantitatively, fundamental natural frequencies and mode shapes were obtained for three different cases of FLM with no cables, one pair of cables, and two pairs of cables in ANSYS and plotted in Figure 9. The natural frequencies obtained were $1.191 \mathrm{~Hz}, 6.244 \mathrm{~Hz}$, and $12.466 \mathrm{~Hz}$, respectively, for these three cases. With the addition of second pair of cables at the decided location, the fundamental natural frequency almost doubles. This provides evidence that the FLM will be significantly stiffened by adding a second pair of cables leading to lower deflections.

\section{Experimental Setup and Validation}

Two experimental setups were constructed: one for finding the natural frequencies of FLM and another for acquiring deflection trajectories at the tip and mid-link. The first experimental setup is shown in Figure 10 where the FLM is rigidly held in a vice. After exciting with a hammer, deflection data at a point on the link is acquired. FFT of the data gives 


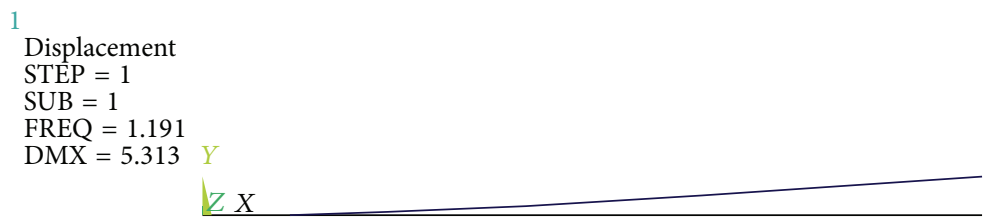

(a) First normal mode of FLM without cables

Displacement

STEP $=1$

SUB $=1$

FREQ $=6.244$

$\mathrm{DMX}=3.968$
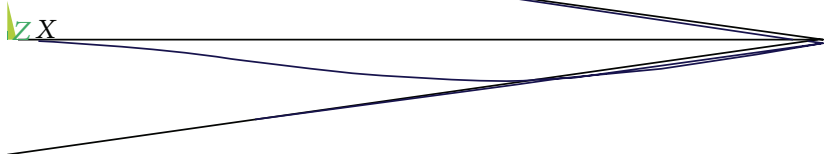

(b) First normal mode of FLM with one pair of cables

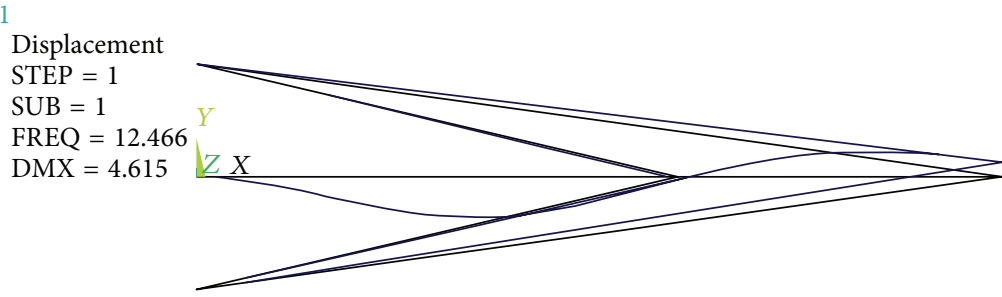

(c) First normal mode of FLM with two pairs of cables

FIgURE 9: Modal analysis in ANSYS.

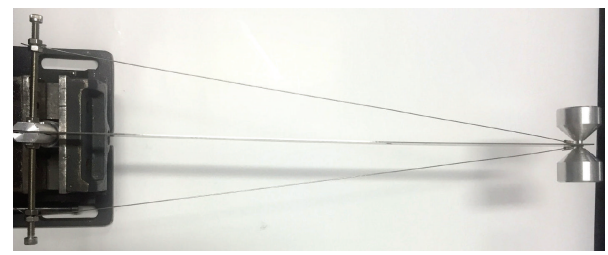

FIGURE 10: Experimental setup for finding natural frequencies for one pair of cables.

the natural frequency. The experiment was repeated for three cases: FLM without cables, FLM with one pair of cables, and FLM with two pairs of cables. The purpose of this experiment is to qualitatively see how much one and two pairs of cables stiffen the FLM.

Figure 11 shows the FFT of FLM deflection data from experiments for three cases. The fundamental natural frequencies obtained are $1.356 \mathrm{~Hz}, 6.66 \mathrm{~Hz}$, and $12.31 \mathrm{~Hz}$, respectively. These fundamental natural frequencies shown by FFT plots closely match those obtained from ANSYS.

The second experimental setup consists of a DC servo motor, power supply, FLM, cables, two displacement sensors, an attachment for mounting displacement sensors, a data acquisition system, and a computer. The DC servo motor is a 100 watt Dynamixel Pro servo H54-100-S500-R which can accept position commands over RS485 communication link from the computer. FLM is mounted on the motor shaft by means of a rigid hub. The attachment for mounting displacement sensors is a rigid manipulator arm attached to the same hub and parallel to the FLM in undeflected condition.

Unlike the setup discussed in Dixit and Prasanth Kumar [27] where deflection is measured only at the end of the trajectory, deflection in local frame can be measured with the current setup throughout the trajectory. The two displacement sensors are noncontact laser based sensors opto-NCDT 1402 with a range of $50 \mathrm{~mm}$ to $150 \mathrm{~mm}$ and a resolution of 10 microns. The sensor outputs are analog which are acquired by a data acquisition system at $1 \mathrm{kHz}$ from which deflection data is obtained. The DC servo motor has digital output pins, one of which is connected to data acquisition system. This output pin, which is usually held low, is triggered to high at the beginning of trajectory command received from computer and held low again from the end of trajectory. The input and output data are time synced using this trigger. The schematic of experimental setup and photograph of cable stiffened FLM are shown in Figures 12(a) and 12(b), respectively. Note that due to difficulty in measuring deflection exactly at the tip where the payload is attached, deflection close to the tip is measured as tip deflection. When comparing with simulation results, deflection of the corresponding point in simulation model is considered tip deflection.

Experiments were conducted with 180 gram payload and deflection at tip and mid-link (where second pair of cables are attached) was measured. Figures 13 and 14 show the simulation and experimental results of FLM deflections with 


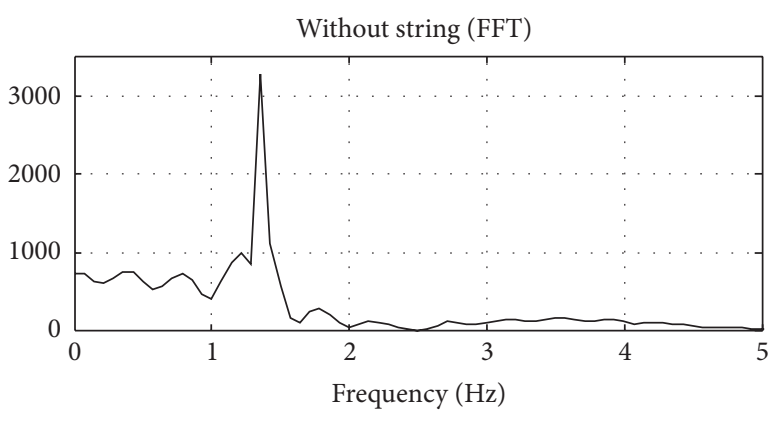

(a) FFT of FLM without cables

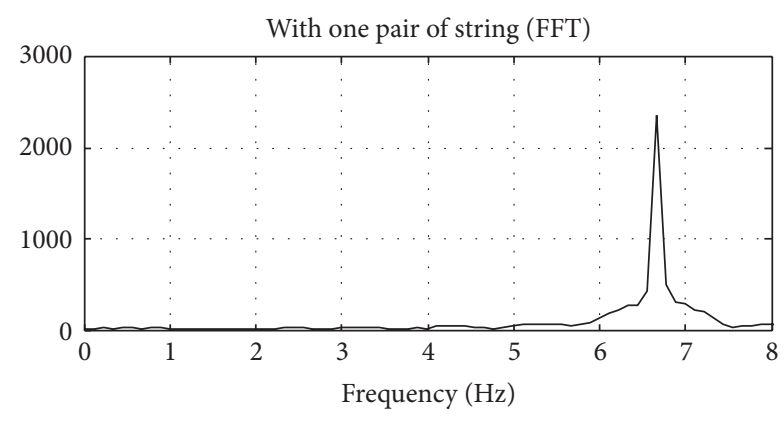

(b) FFT of FLM with one pair of cables

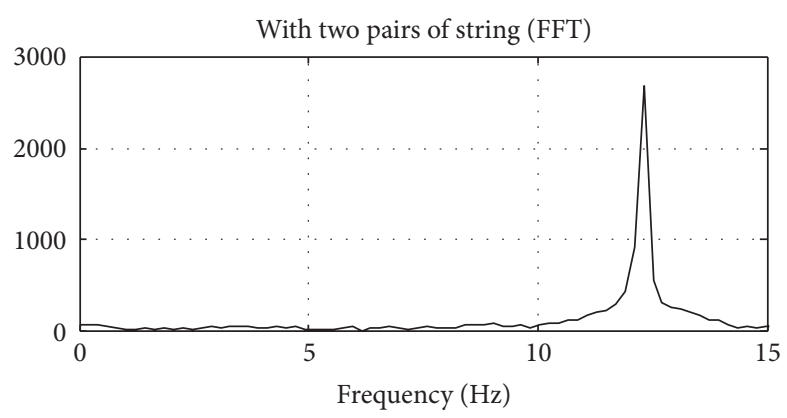

(c) FFT of FLM with two pairs of cables

FIGURE 11: FFT of FLM deflection data for three cases.

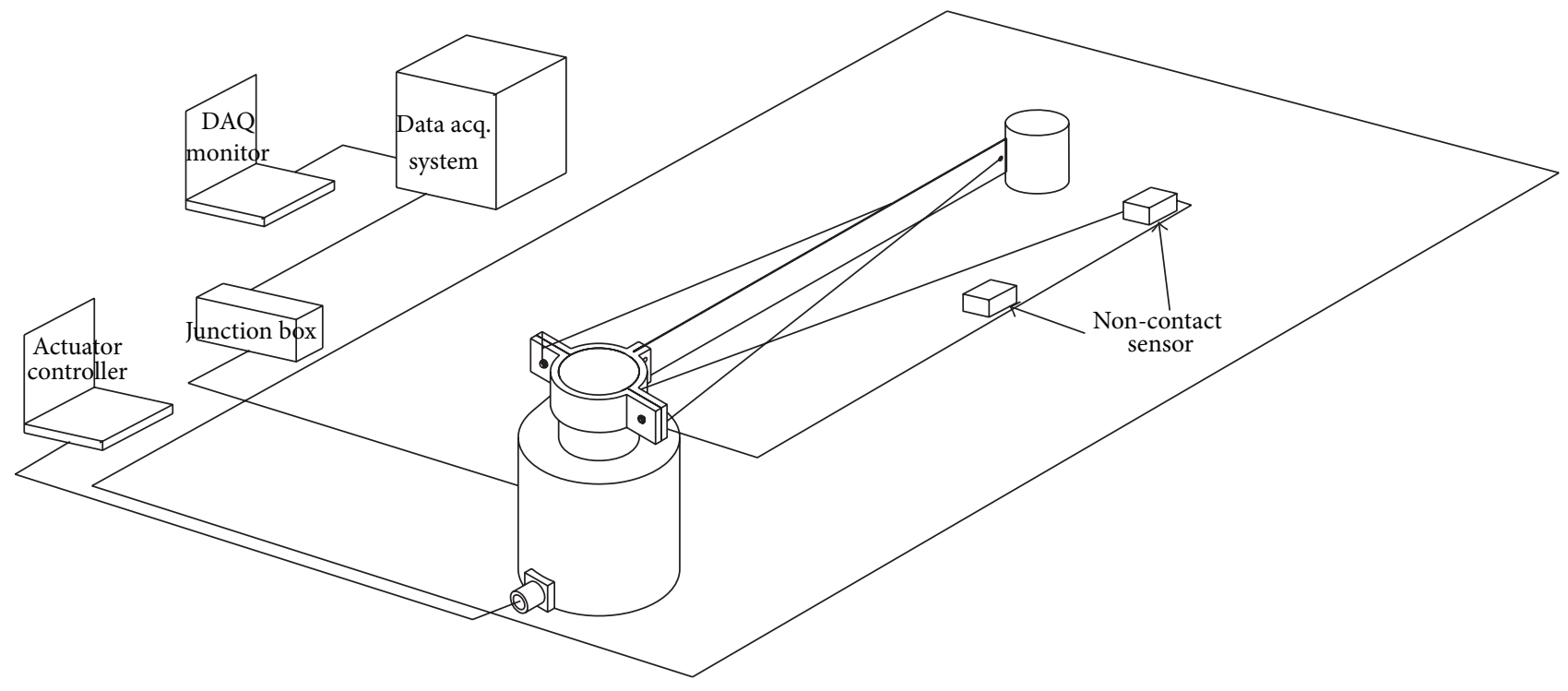

(a) Schematic of experimental setup of cable stiffened FLM

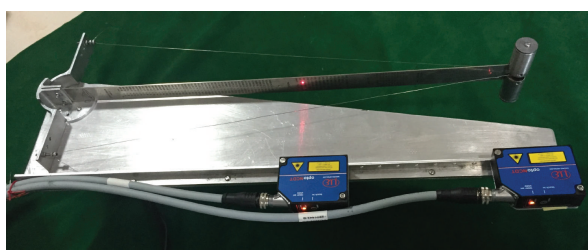

(b) Experimental setup for one pair of cables

FIGURE 12: Experimental setup of cable stiffened FLM. 


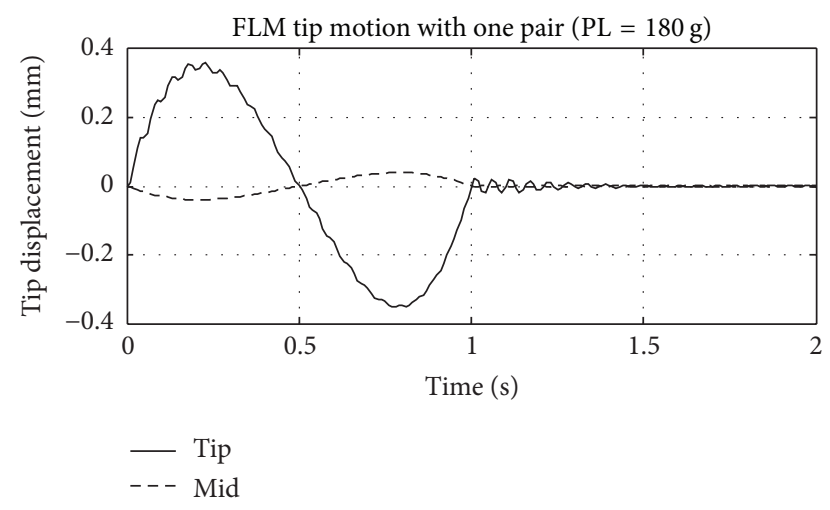

FIGURE 13: Simulation results of tip and mid-link deflections for one pair of cables.

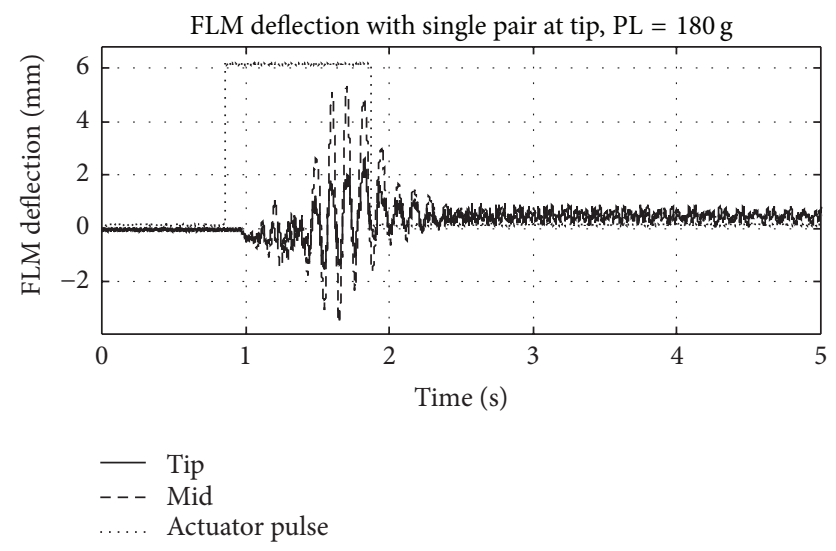

FIGURE 14: Experimental results of tip and mid-link deflections for one pair of cables.

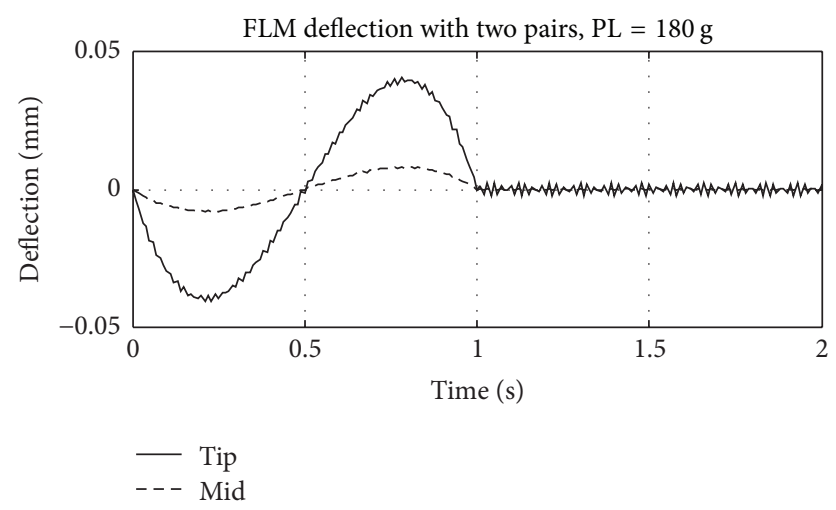

FIGURE 15: Simulation results of tip and mid-link deflections for two pairs of cables.

one pair of cables attached at the tip, respectively. Similarly, Figures 15 and 16 show the simulation and experimental results of FLM deflections with two pairs of cables, respectively. The actuator pulse shown in experimental results indicate the duration of trajectory command.

Comparing the simulation and experimental results, it is clear that addition of second pair of cables in fact reduces

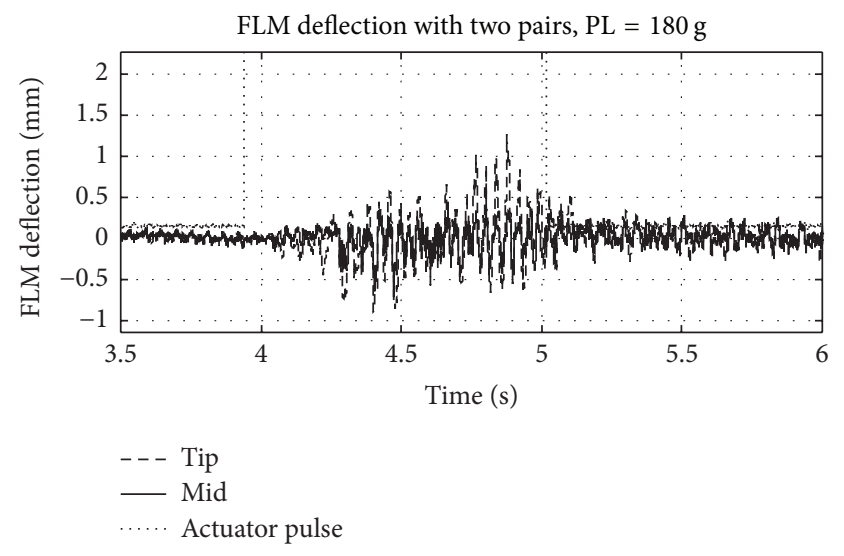

FIGURE 16: Experimental results of tip and mid-link deflections for two pairs of cables.

the deflection at both the tip and the mid-link. However, deflections occurring during experiments are about one order of magnitude higher than those of simulation results and higher frequency components also appear. As a first guess, we believed that the discrepancy was due to the presence of slackness in the cables allowing the deflections to increase. Even when we tried to reduce the slackness by introducing a small pretension in the cables, results were similar. Another possibility is the presence of small bends in the steel cables connecting the link to the rigid hub. These bends act as curved beams with low bending stiffness compared to the axial stiffness of the cable. We theorize that when the payload is subjected to inertia forces during the link motion, low stiffness, till the bend opens up completely, causes connected points on the link to deflect significantly. This is a major limitation of cable stiffening method.

\section{Conclusions}

It is known that any flexible body has infinity degrees of freedom. Therefore, ideally a single input at the hub will not be able to track arbitrary trajectories of FLMs making them underactuated systems. As the interest is to restrict oscillations of the tip alone, the methodology presented in this paper attempts to restrict DOFs of the tip by attachment of cables. A systematic way is presented for the attachment of cables to minimize link deflections. Foreshortening effect due to mid-link deflection gets minimized by attachment of additional pairs of cables at the specified locations. Two sets of experimental setups were constructed. It is concluded that the simulation results and experimental results have very good agreement as far as the fundamental natural frequencies are concerned.

Trajectories obtained from second experimental setup show one order of magnitude discrepancy with simulation results, although the trend of reduced deflection with increase in the number of pairs of cables is present. The major limitation of large deflections found in experiments may be overcome by more accurate fabrication and assembly of cables on the FLM. 


\section{Competing Interests}

The authors declare that there is no conflict of interests regarding the publication of this paper.

\section{Acknowledgments}

This work was supported by the project "Cable Stiffened Flexible Link Manipulator for Pick-and-Place Tasks” (YSS/2014/ 000010) sponsored by Department of Science and Technology, Government of India.

\section{References}

[1] R. H. Cannon Jr. and E. Schmitz, "Initial experiments on the end-point control of a flexible one-link robot," The International Journal of Robotics Research, vol. 3, no. 3, pp. 62-75, 1984.

[2] W. D. Zhu and C. D. Mote Jr., "Dynamic modeling and optimal control of rotating Euler-Bernoulli beams," Journal of Dynamic Systems, Measurement and Control, vol. 119, no. 4, pp. 802-808, 1997.

[3] M. H. Korayem, H. N. Rahimi, and A. Nikoobin, "Mathematical modeling and trajectory planning of mobile manipulators with flexible links and joints," Applied Mathematical Modelling, vol. 36, no. 7, pp. 3229-3244, 2012.

[4] W. Chen, "Dynamic modeling of multi-link flexible robotic manipulators," Computers \& Structures, vol. 79, no. 2, pp. 183195, 2001.

[5] A. Fenili and J. M. Balthazar, "The rigid-flexible nonlinear robotic manipulator: modeling and control," Communications in Nonlinear Science and Numerical Simulation, vol. 16, no. 5, pp. 2332-2341, 2011.

[6] M. Khairudin, "Dynamic modelling of a flexible link manipulator robot using AMM," TELKOMNIKA Indonesian Journal of Electrical Engineering, vol. 6, no. 3, pp. 185-190, 2008.

[7] S. Bošnjak and N. Zrnić, "On the dynamic modelling of exible manipulators," FME Transactions, vol. 34, pp. 231-237, 2006.

[8] A. A. Ata, E. H. Haraz, A. E. A. Rizk, and S. N. Hanna, "Kinematic analysis of a single link flexible manipulator," in Proceedings of the IEEE International Conference on Industrial Technology (ICIT '12), pp. 852-857, Athens, Greece, March 2012.

[9] J.-C. Piedboeuf, "Six methods to model a flexible beam rotating in the vertical plane," in Proceedings of the IEEE International Conference on Robotics and Automation (ICRA '01), vol. 3, pp. 2832-2839, IEEE, Seoul, Republic of Korea, May 2001.

[10] H. Sugiyama, N. Kobayashi, and Y. Komaki, "Modeling and experimental methods for dynamic analysis of the spaghetti problem," Journal of Vibration and Acoustics, vol. 127, no. 1, pp. 44-51, 2005.

[11] G. S. Kiran, A. Kumar, P. M. Pathak, and N. Sukavanam, "Trajectory control of flexible space robot," in Proceedings of the IEEE International Conference on Mechatronics and Automation (ICMA '08), pp. 738-743, IEEE, Takamatsu, Japan, August 2008.

[12] I. A. Mahmood, S. O. R. Moheimani, and B. Bhikkaji, "Precise tip positioning of a flexible manipulator using resonant control," IEEE/ASME Transactions on Mechatronics, vol. 13, no. 2, pp. 180-186, 2008.

[13] V. Etxebarria, A. Sanz, and I. Lizarraga, "Control of a lightweight flexible robotic arm using sliding modes," International Journal of Advanced Robotics, vol. 2, no. 2, pp. 103-110, 2005.
[14] G. Mamani, J. M. A.-D. Silva, and V. Feliu-Batlle, "Least squares state estimator based sliding mode control of a very lightweight single-link flexible robot arm," in Proceedings of the IEEE 2009 International Conference on Mechatronics (ICM '09), pp. 1-6, IEEE, Malaga, Spain, April 2009.

[15] R. Fareh, M. Saad, and M. Saad, "Adaptive control for a single flexible link manipulator using sliding mode technique," in Proceedings of the 6th International Multi-Conference on Systems, Signals and Devices (SSD '09), pp. 1-6, IEEE, Djerba, Tunisia, March 2009.

[16] S. Kurode and P. Dixit, "Output feedback control of flexible link manipulator using sliding modes," in Proceedings of the 2012 7th International Conference on Electrical and Computer Engineering (ICECE '12), pp. 949-952, IEEE, Dhaka, Bangladesh, December 2012.

[17] S. S. Ge, T. H. Lee, and G. Zhu, "A nonlinear feedback controller for a single-link flexible manipulator based on a finite element model," Journal of Robotic Systems, vol. 14, no. 3, pp. 165-178, 1997.

[18] R. Morales, V. Feliu, and V. Jaramillo, "Position control of very lightweight single-link flexible arms with large payload variations by using disturbance observers," Robotics and Autonomous Systems, vol. 60, no. 4, pp. 532-547, 2012.

[19] M. Baroudi, M. Saad, and W. Ghie, "State-feedback and linear quadratic regulator applied to a single-link flexible manipulator," in Proceedings of the 2009 IEEE International Conference on Robotics and Biomimetics (ROBIO '09), pp. 1381-1386, IEEE, Guilin, China, December 2009.

[20] M. A. Ahmad and Z. Mohamed, "Techniques of vibration and end-point trajectory control of flexible manipulator," in Proceedings of the 6th International Symposium on Mechatronics and its Applications (ISMA '09), pp. 1-6, IEEE, Sharjah, United Arab Emirates, March 2009.

[21] H. Yang, M. H. Ang Jr., and H. Krishnan, "Control of a tiploaded flexible-link robot using shaped input command," in Proceedings of the American Control Conference (ACC '98), vol. 5, pp. 3075-3076, IEEE, Philadelphia, Pa, USA, June 1998.

[22] W. Chatlatanagulchai, V. M. Beazel, and P. H. Meckl, "Command shaping applied to a flexible robot with configurationdependent resonance," in Proceedings of the American Control Conference, IEEE, Minneapolis, Minn, USA, June 2006.

[23] K.-P. Liu and Y.-C. Li, "Vibration suppression for a class of flexible manipulator control with input shaping technique," in Proceedings of the International Conference on Machine Learning and Cybernetics, pp. 835-839, August 2006.

[24] K.-P. Liu, "Experimental evaluation of preshaped inputs to reduce vibration for flexible manipulator," in Proceedings of the 6th International Conference on Machine Learning and Cybernetics (ICMLC '07), vol. 4, pp. 2411-2415, IEEE, Hong Kong, August 2007.

[25] M. Romano, B. N. Agrawal, and F. Bernelli-Zazzera, "Experiments on command shaping control of a manipulator with flexible links," Journal of Guidance, Control, and Dynamics, vol. 25, no. 2, pp. 232-239, 2002.

[26] N. Seth and K. S. Rattan, "Vibration control of flexible manipulators," in Proceedings of the IEEE National Aerospace and Electronics Conference (NAECON '92), pp. 876-882, 1992.

[27] R. Dixit and R. Prasanth Kumar, "Cable stiffened flexible link manipulator," in Proceedings of the IEEE/RSJ International Conference on Intelligent Robots and Systems (IROS '14), pp. 871876, Chicago, Ill, USA, September 2014. 


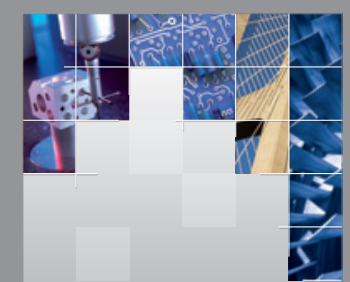

\section{Enfincering}
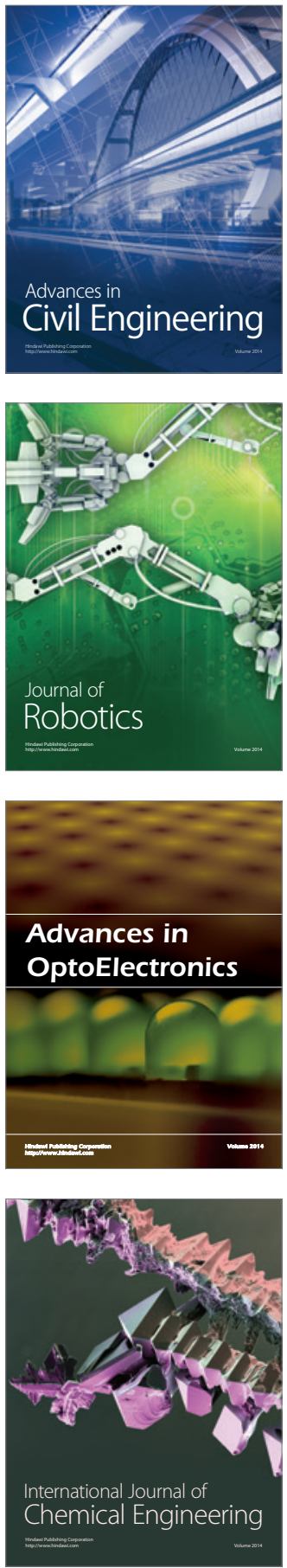

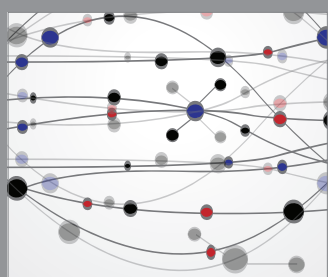

The Scientific World Journal

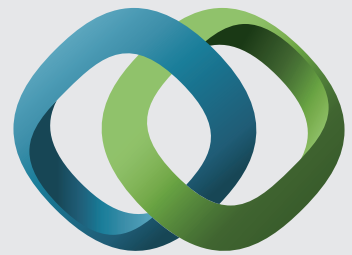

\section{Hindawi}

Submit your manuscripts at

http://www.hindawi.com
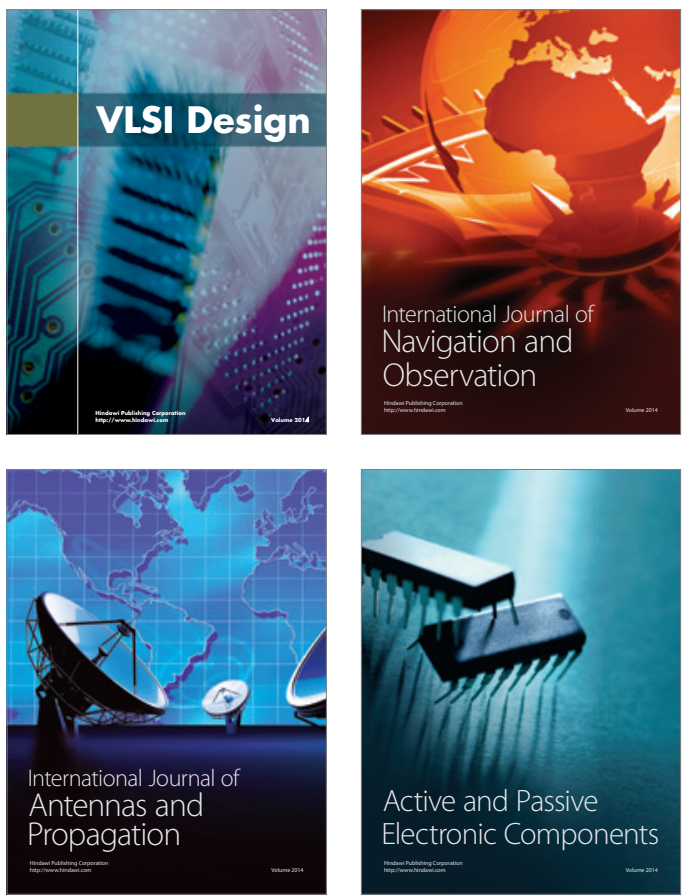
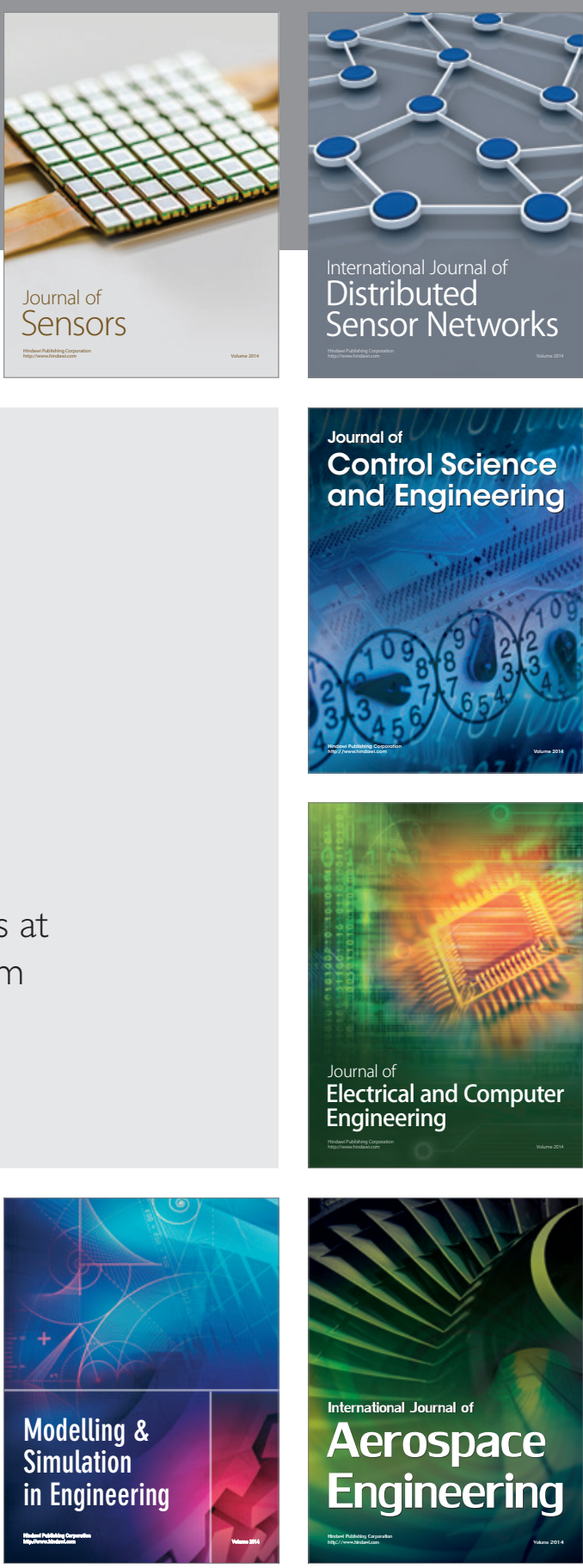

International Journal of

Distributed

Sensor Networks

Journal of

Control Science

and Engineering
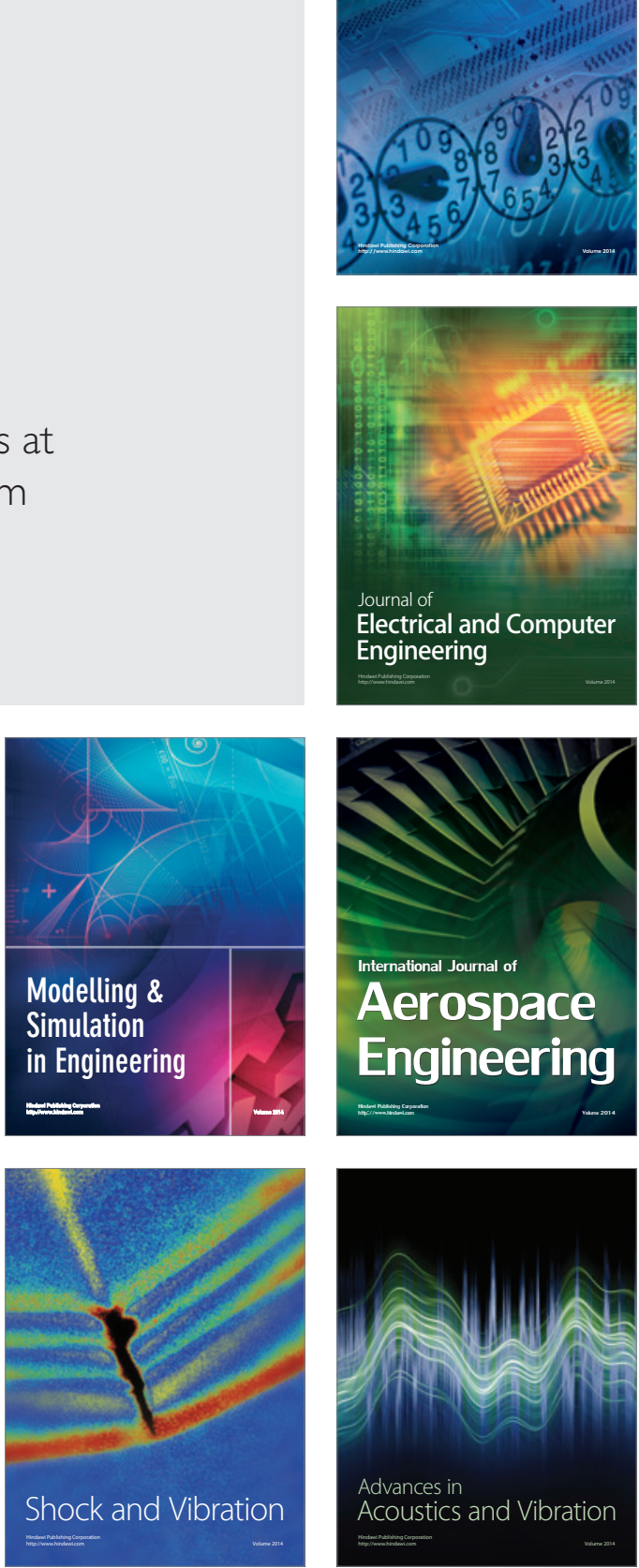